(C)2006 IEEE. Personal use of this material is permitted. However, permission to reprint/republish this material for advertising or promotional purposes or for creating new collective works for resale or redistribution to servers or lists, or to reuse any copyrighted component of this work in other works must be obtained from the IEEE. 


\title{
AN AUTONOMOUS, LOW COST, DISTRIBUTED METHOD FOR OBSERVING VEHICLE TRACK INTERACTIONS
}

\author{
Peter J Wolfs PhD C.P.Eng, Steven Bleakley B.E., Steven T Senini PhD and Peter Thomas PhD \\ Centre for Railway Engineering, \\ Central Queensland University, \\ Rockhampton, 4702 Queensland, Australia. \\ Tel: (+61) 7-49309599; Fax (+61) 7-49306732 \\ Email:p.wolfs@cqu.edu.au
}

\begin{abstract}
Experience and field studies have shown that track geometry alone is not a good predictor of rail vehicle response. This paper describes a family of "Health Card" devices - an autonomous device that can be distributed on rolling stock to analyse the vehicle responses. As a distributed system is desired, and the intent is to apply this technology widely across a vehicle fleet, a low initial capital cost and low operating cost solution is desirable. As a consequence the Health Card performs all its sensing operations on the car body and avoids the costs and complications of sensing below the car body especially on unsprung components. Health Cards use solid-state transducers including accelerometers and angular rate sensors with a coordinate transform to resolve car body motions into six degrees of freedom. They then apply spectrogram techniques to obtain a time-frequency representation of the car body motion. These representations are autonomously analyzed to detect and classify transient dynamic events and to infer track degradation or operational risks.
\end{abstract}

\section{INTRODUCTION}

Internationally the requirement for track maintenance is determined using specialized track geometry measurement vehicles to determine track condition. For example, track irregularity tolerance bands are specified for the Australian defined interstate rail network in the Department of Transport and Regional Services publication DTRS Volume 4, Part 3, [1].

The complex interaction between various vehicle types and the track means that acceptable track irregularity levels can be difficult to determine. Predicting vehicle responses online from track measurement data has been addressed internationally by various means. Esveld outlines a multiple input single output transfer function based system used in the Netherlands named Vehicle Response Analysis (VRA), [2]. Simplified vehicle dynamics models have been usefully applied to highlight potential derailment sites for specific vehicle types, [3]. One system in the United States uses artificial neural networks to predict vehicle reactions from track measurements, [4].

Track condition monitoring via the use of instrumented vehicles has been applied widely and various systems are available internationally. Freight wagon instrumentation studies have shown that severe dynamic forces occur when the irregularity wavelength and train speed combine to excite a resonant mode in the vehicle, [5-7]. An "Autonomous Ride Monitoring System" (ARMS) developed by Amtrak, [8], monitors peak and RMS acceleration on the $10 \mathrm{~Hz}$ low pass filtered signal in accordance with standard requirements outlined by the FRA, [9]. RideMon ${ }^{\mathrm{TM}}$ by UK based AEA Technology Rail is an equivalent system, [10]. A multiple vehicle monitoring system for in revenue service applications has also been proposed but is rather more expensive than the solution proposed by this paper, [11].

Vehicle condition monitoring is also beneficial. A recent study by a research team at the Technical University of Berlin focused on dangerous goods wagon derailment protection and involved purposely derailing an instrumented wagon on numerous occasions and at incremental speeds of up to $40 \mathrm{~km} / \mathrm{h}$. It was shown that accelerometers placed on the body of a tank car are very effective in detecting a derailment, [12]. Derailment of one or two wheel sets can remain unnoticed for long distances, in some cases tens of kilometres, causing significant damage to infrastructure.

Many commercial systems can recover detailed data recordings for offline user applications by storing and transmitting the time series information to central databases and geographical information systems. This functionality requires data storage and communications capacities that have price impacts. A lower cost solution is to perform waveform analysis autonomously at the monitoring device so that only event codes are transmitted.

This paper presents a novel device for the online analysis of car body motion signals to detect track condition and provide 
derailment monitoring. Two solutions are presented - a wired solution and wireless solution.

The wireless solution is a solar powered radio based device and is intended for applications where a train-wire is not available. This includes the majority of existing freight operations. Low power spread spectrum radios are used to establish a distributed communications network along the train. Car body motion signals are converted into a timefrequency representation so that events can be detected according to their short-term "modal" content content. The modal content relates to the natural vibration modes of the vehicle system such as bounce roll and pitch behaviors.

The wired solution uses a two conductor train-line to distribute power to the Health Cards and to provide a communications channel.

The structure of the paper may be described in four parts. Firstly, a brief description of the health card hardware is given. Secondly, an understanding of the signals under measurement is established and time-frequency analysis is explained. Thirdly, the signal detection scheme is introduced. Finally some field and vehicle testing laboratory results are presented.

\section{HEALTH CARD HARDWARE}

Two variations of the Health Card hardware have been produced. The radio based system focuses on applications where power and communications are not distributed to the cars on Australian freight trains. The wireless Health Card is solar powered. The first generation of the Health Card achieves a total consumption of $400 \mathrm{~mW}$ or an energy requirement of $9.6 \mathrm{Wh}$ daily. This is provided by a $10 \mathrm{Wp}$ solar panel, which under the best orientations could produce $60 \mathrm{Wh}$ daily in the Central Queensland region. Variable orientations due to car body motion, fouling by dirt and other contaminants and weather require a significant margin of excess capacity. The wireless Health Card has an $80 \mathrm{Wh}$ lithium ion battery that provides better than eight days autonomy in the absence of any solar input.

The major consumer of power is the processor, a Rabbit 3000 processor that requires $200 \mathrm{~mW}$ operating at $40 \mathrm{MHz}$. The current consumption due to processing is directly proportional to clock frequency. The requirement to minimize power consumed by the processor means that both sampling rates and computation time required by the algorithms must be minimized. The Rabbit 3000 processor currently used in the prototype system has $256 \mathrm{~KB}$ of RAM of which $64 \mathrm{~KB}$ is efficiently accessible.

The other major power consumer is the radio system, each card uses a pair of blue tooth radios to communicate across the buffers to the adjacent cars. The communications system must operate in a daisy chain topology as represented in Figure 1. This topology generates increasing traffic for nodes near the locomotive with increasing rake length. Clearly data transmissions from the nodes must be limited.

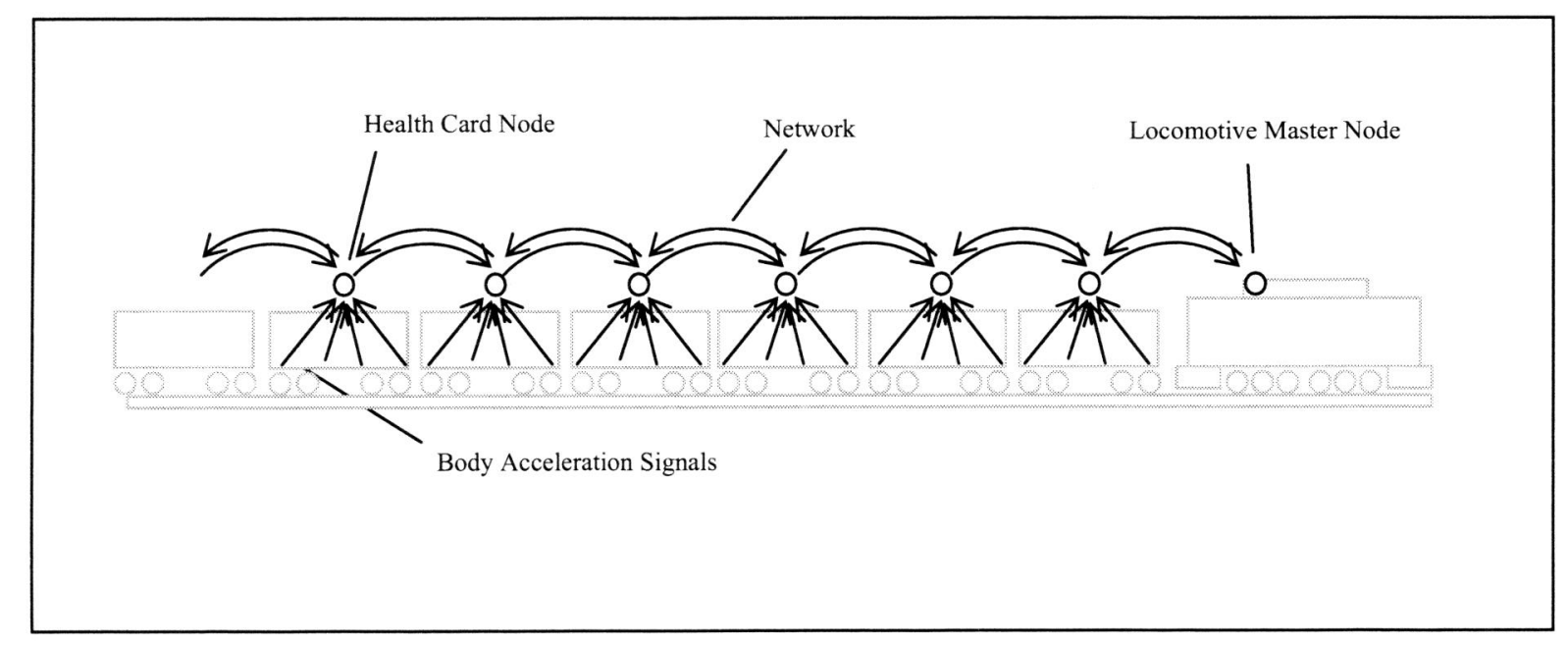

Figure 1. Communications System Network Topology

The radio based health card utilises the Analog Devices ADXL202/10 dual axis acceleration sensor to detect body motion. This sensor is preferable because of its low cost and power use, and relative ease of installation compared to strain gauges. The ADXL202/10 measures acceleration in two perpendicular axes and is capable of sensing frequencies from DC to several kilohertz. To secure the full six degrees of freedom for the car body motions two or three axis accelerometers are placed at three corners of the car body. By the application of a co-ordinate transformation, these signals can be converted into longitudinal, lateral and vertical accelerations as well as pitch roll and yaw. Figure 2 shows a wireless Health Card set, without solar cells, placed on a bench top for testing. Each set is temporarily mounted on a board. The health card is visible mounted centrally on the board with three diecast boxes on the board corners which contain accelerometers and the radios. 


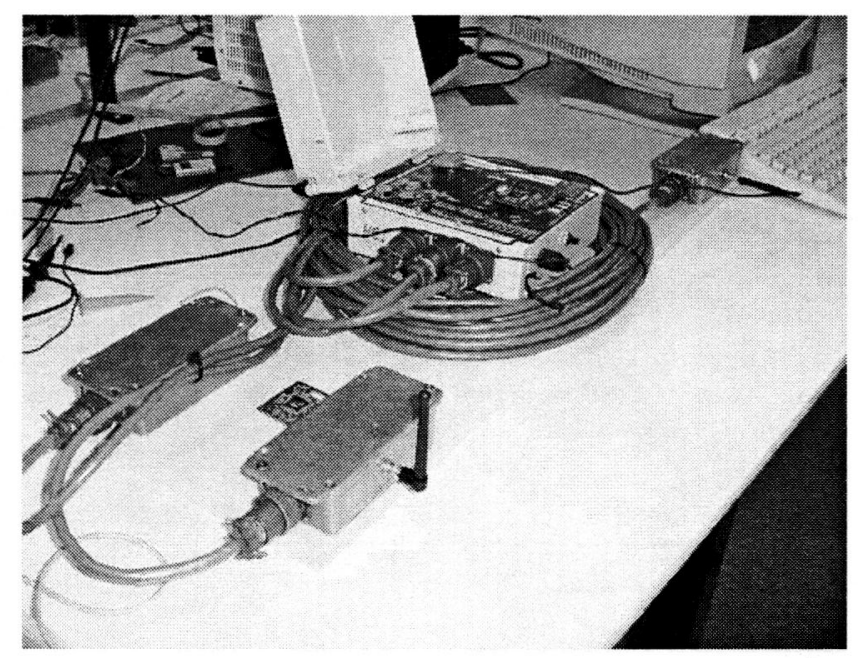

Figure 2. Wireless Prototype System Bench Test Arrangement

The train-wire based health card utilizes a two conductor train wire. Power is distributed at $240 \mathrm{Vdc}$ and the same two conductors are used to establish data communications using Echelon Corporation power line transceiver devices, [12]. These devices utilise the IEEE Standard for Communications Protocol Aboard Trains, 1473-1999, and this is often referred to as the Lonworks $\left({ }^{\circ}\right.$ protocol. This protocol is used for Electronically Controlled Pneumatic (ECP) brake systems. A wire based health card is shown in Figure 3. This prototype utilizes accelerometers to detect the three translational accelerations of the car body. Analog Devices angular rate sensors, ADXRS150, are used to determine the car body rotations without the need for placing accelerometers at the car body corners. As power is more abundantly available, the wire based card splits the communications tasks and the signal analysis tasks between two processors. Communications is handled by an Echelon Corporation Neuron device and the signal processing is performed by a Texas Instruments TMS320C2812. In comparison to the solar powered radio based card, the train-wire based card is capable of more advanced local signal processing. This paper will focus on one processing approach - spectrograph analysis - which both Health Card systems can perform.

\section{HEALTH CARD SIGNAL PROCESSING}

The Health Card system relies on accelerometer or angular rate inputs as the primary source of car body motion detection. When inspecting acceleration signals in the time domain, it must be understood that the magnitude of the acceleration signal is proportional to the square of the instantaneous frequency of the displacement signal.

$$
|\mathrm{A}(\omega)| \propto \omega^{2}
$$

Signals that relate to large motions (displacements) are often much lower frequency than the surrounding high frequency vibrations, yet the high frequency vibrations have a larger acceleration magnitude. Angular rate signals show similar behaviours but the dependence is only proportional to frequency. As a precursor to the Health Card signal processing

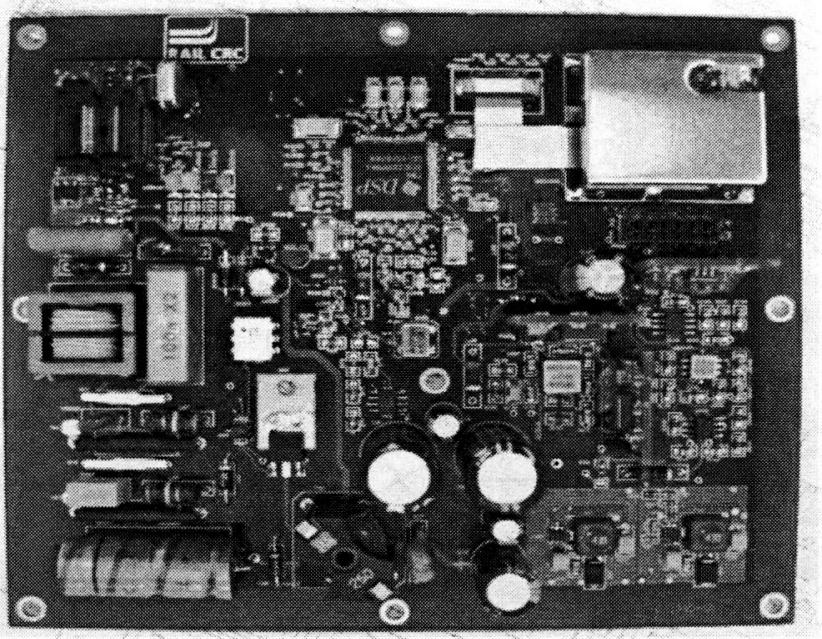

Figure 3. Train-wire based Health Card Prototype.
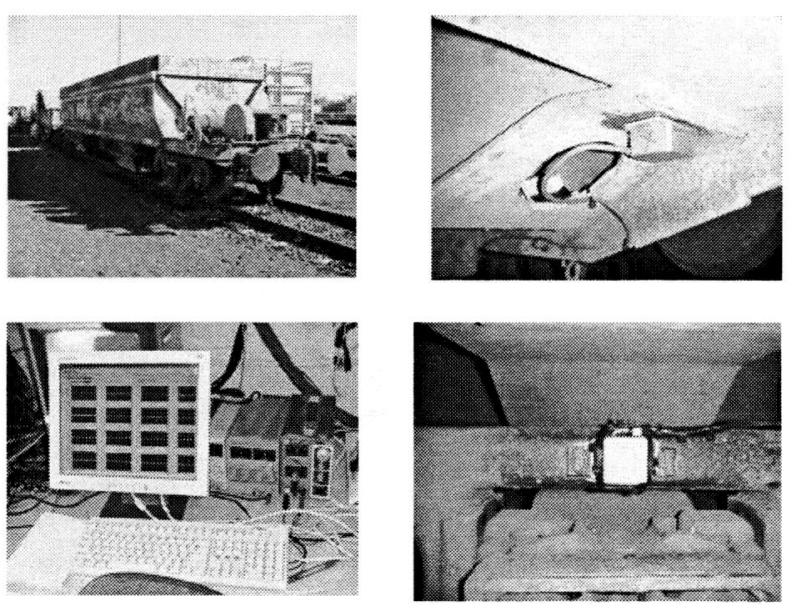

Figure 4. Field data acquisition. Clockwise from top leftBallast Wagon; Body Mounted Accelerometer; Labview System, Side Frame mounted Accelerometer.

system design, car body acceleration signatures where captured using a gondola ballast wagon as shown in Figure 4. Figure 5 shows an example of an acceleration signal in the vertical axis collected from the front left corner when viewed facing in the direction of travel. The marked sections are the signals caused by rough ride events that were noticed by the operators during the data acquisition. Figure 5 shows the results of filtering the signal with a first order filter with a corner frequency of $10 \mathrm{~Hz}$. The dominant high frequency amplitudes are reduced but the signal to noise ratio is still poor due to the $\omega^{2}$ relationship which still applies within the 0 $-10 \mathrm{~Hz}$ range of the signal. An alternative to a simple time domain solution based on filtering and applying a threshold is to analyze the $0-10 \mathrm{~Hz}$ signal range in the frequency domain.

The Health Card system used the short term Fourier Transform to generate a spectrogram of the wagon body accelerations. Other methods of generating spectrograms are possible, such as a range of wavelet transforms, but these do 
not appear to offer strong advantages. In fact, given that vehicle dynamics packages such as VAMPIRE ${ }^{\circledR}$ will explicitly identify the dominant vibratory modes of a vehicle, a strong case exists for a traditional Fourier approach which generates outputs that are readily interpreted by a vehicle dynamic expert. Applying the short term FFT method yields the spectrogram shown in Figure 6. In the spectrogram image, the vertical axis is increasing frequency, and the horizontal axis is time. The dark patches at 16 seconds and 25 seconds are caused by the dynamics that were observed during the acquisition. The transient observed at 29 seconds is far less pronounced in the spectrogram. An autonomous method is desired to detect events such as those revealed by the spectrogram representation in Figure 6, while allowing scope to further analyze the signal in order to discern different types of events. Applying a sliding $N$ point FFT allows the $0-10 \mathrm{~Hz}$ range to be represented as a set of coefficients calculated over a finite time $\Delta \mathrm{t}=N T_{\mathrm{s}}$ where $T_{\mathrm{s}}$ is the sampling period. For each $\Delta t$, the coefficients can be considered as axes of a signal space. The concept can be visualized in three dimensions (3D) by dividing the $0-10 \mathrm{~Hz}$ range into three frequency bands, $\mathrm{x}, \mathrm{y}$ and $\mathrm{z}$. The $N$ point FFT result for each time slot is plotted as a point in a $3 \mathrm{D}$ space as illustrated in Figure 7. The data used is from the time signal of Figure 5.

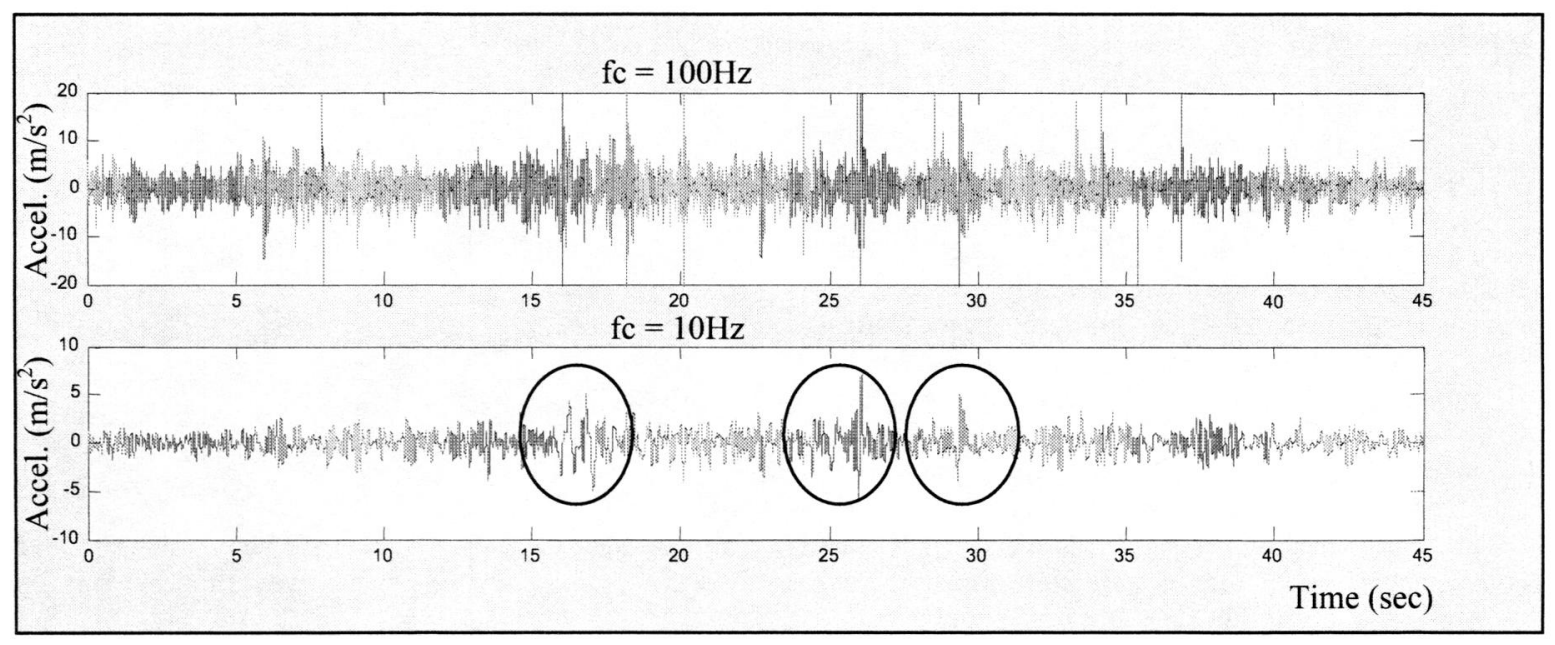

Figure 5. Car Body Acceleration $100 \mathrm{~Hz}$ Filtered (top) and 10Hz Filtered (bottom). (Signals from noticeable events circled)

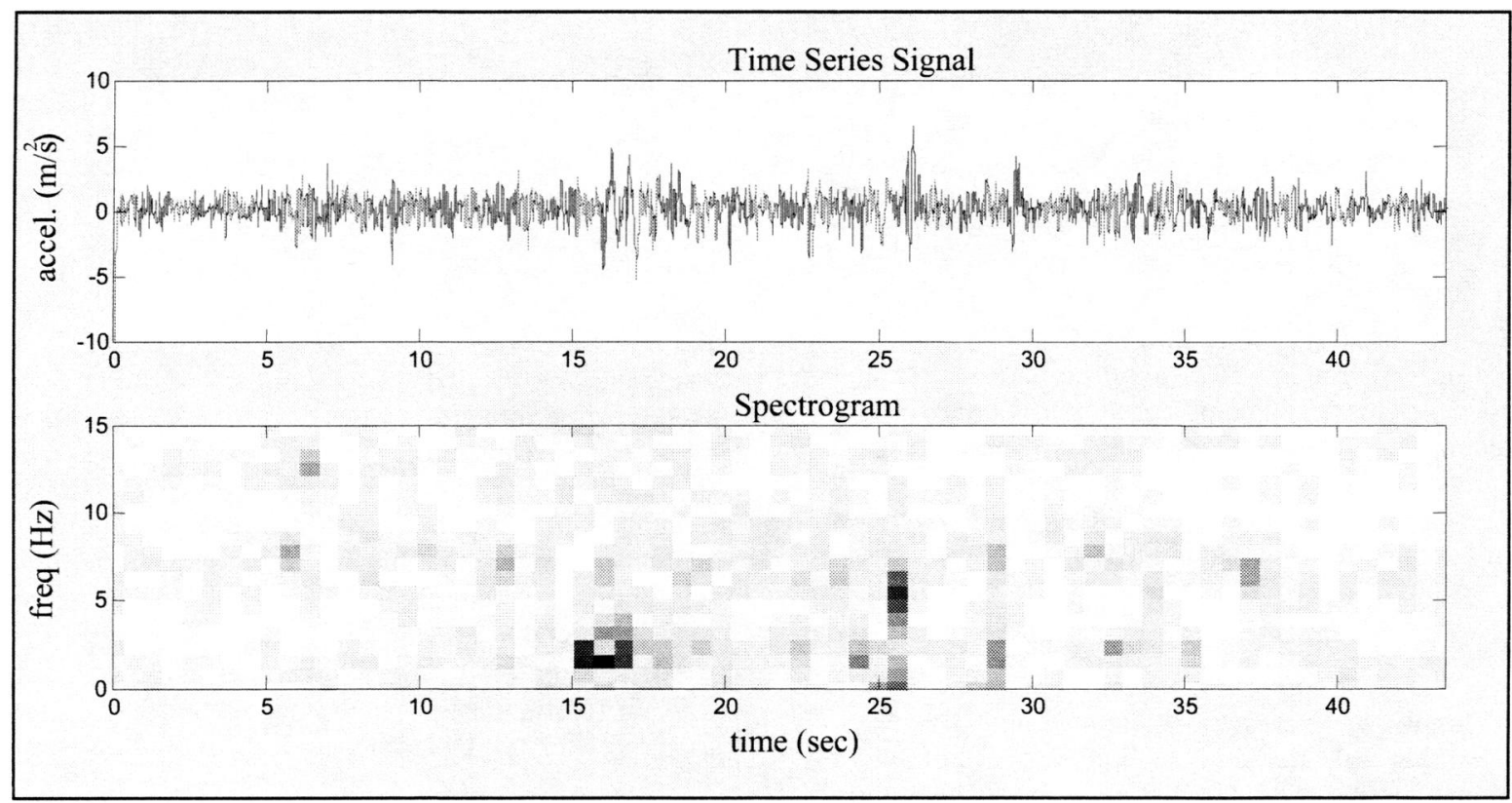

Figure 6. Time Series (top) and Spectrogram (bottom) of Car Body Acceleration with Dynamic Events at 16, 25 and 29 seconds. 
Abnormal events can be detected by defining a region in the $3 \mathrm{D}$ space and identifying points outside of this region. The simplest surface to define is a sphere with radius $d_{\mathrm{T}}$. The distance to each point is given by Equation (2).

$d_{\mathrm{n}}^{2}=\mathrm{x}_{\mathrm{n}}^{2}+\mathrm{y}_{\mathrm{n}}^{2}+\mathrm{z}_{\mathrm{n}}^{2}$

If $d_{\mathrm{n}}{ }^{2}>d_{\mathrm{T}}{ }^{2}$, the $\mathrm{n}^{\text {th }}$ time interval is flagged as a detected event. The power spectrum is, conveniently, the magnitude squared of the FFT coefficients, so the distance can be calculated by simply summing the power spectrum coefficients. The normal space can be changed to distinguish between different types of events. The signal can be weighted with constant coefficients $\mathrm{C}_{1}, \mathrm{C}_{2}$ and $\mathrm{C}_{3}$ to effectively distort the decision boundary in relation to the signal distribution, resulting in Equation 3.

$$
d_{\mathrm{n}}^{2}=\mathrm{C}_{1} \mathrm{x}_{\mathrm{n}}^{2}+\mathrm{C}_{2} \mathrm{y}_{\mathrm{n}}^{2}+\mathrm{C}_{3} \mathrm{z}_{\mathrm{n}}^{2}
$$

To resolve the various oscillation modes of the wagon system, a higher frequency resolution than used to illustrate the algorithm in Figure 6 is required. The algorithm presented in the results section uses 13 coefficients to cover the $0-10 \mathrm{~Hz}$ frequency range. The output is the distance squared $d_{\mathrm{n}}^{2}$ from the origin according to Equation (4).

$$
d_{\mathrm{n}}{ }^{2}=\mathrm{C}_{1} \mathrm{~A}_{\mathrm{n}}\left(\mathrm{f}_{1}\right)^{2}+\mathrm{C}_{2} \mathrm{~A}_{\mathrm{n}}\left(\mathrm{f}_{2}\right)^{2}+\ldots+\mathrm{C}_{13} \mathrm{~A}_{\mathrm{n}}\left(\mathrm{f}_{13}\right)^{2}
$$

Figure 8 shows the results of the algorithm applied to the dynamic event. The time series signal is low pass filtered at $10 \mathrm{~Hz}$ and sampled at 200 samples per second. An overlapping 256 point FFT is taken every 128 points with a 256 point Hanning window, so that a short term spectrum is produced every 0.64 seconds. The top signal is the time series data. The second plot is the spectrogram of the signal. The bottom plot is the classifier output variable calculated by Equation 4 with $\mathrm{C}_{\mathrm{k}}=1$ for all $\mathrm{k}$.

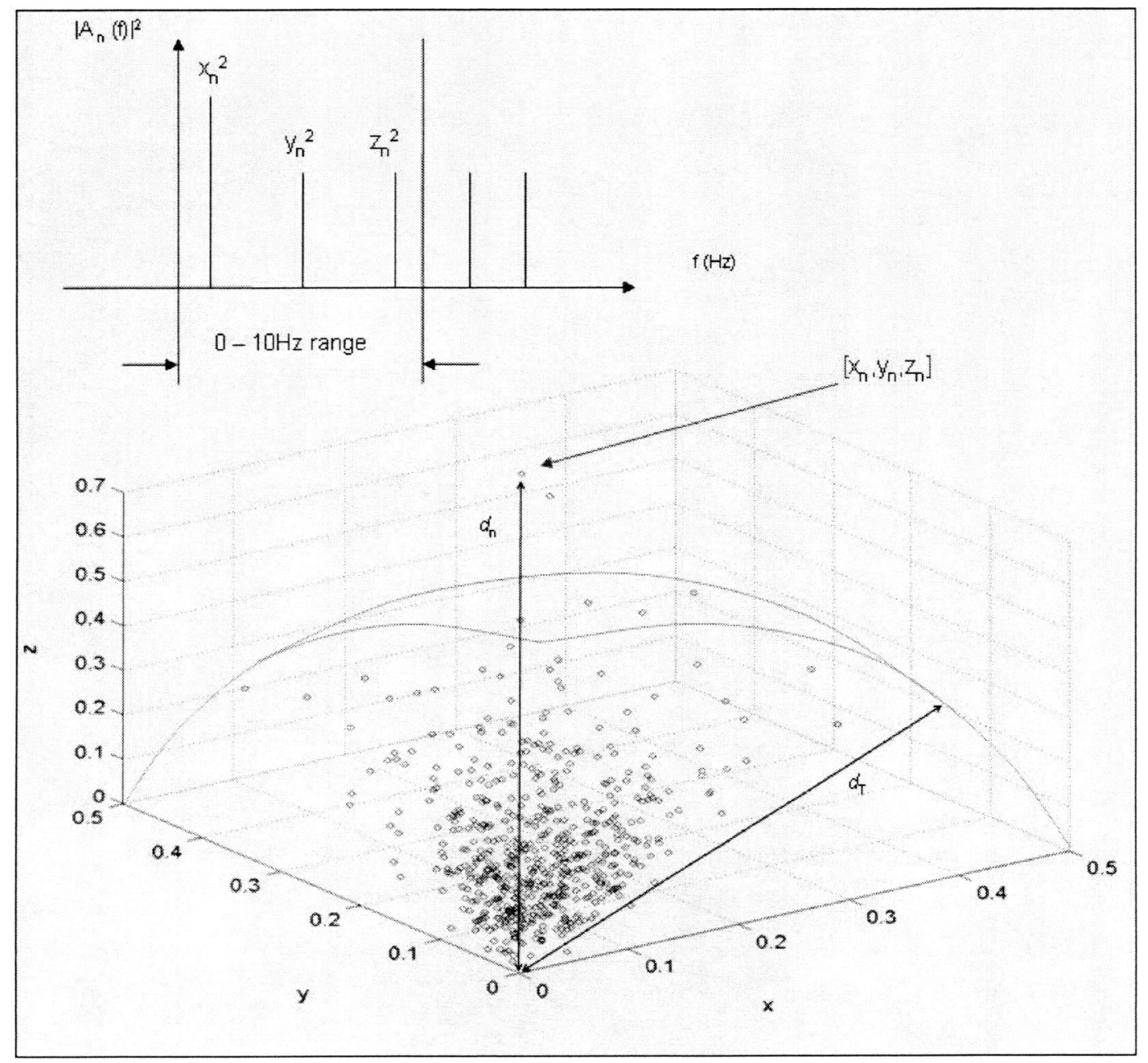

Figure 7. Event Detection Concept using Three Coefficients Defining a Spherical Threshold.

Comparing the time series waveform (top) with the classifier output (bottom) there is a large improvement of signal to noise ratio of approximately $5 / 4$ to $4 / 2$ and a $128: 1$ data reduction from 200 samples per second to 1.56 samples per second. A threshold at $d_{\mathrm{T}}^{2}=3 \times 10^{4}$ would detect a dynamic event at 16, 25 and 29 seconds. 
The coefficients in the spectrogram show clear differences in the frequency content between each detected events. While this spectrogram only relates to one wagon body accelerometer, and the rotational and translational modes are not separable with one device, the transient at 16 seconds has a frequency that is characteristic of the vertical bounce mode. In the Health Card system it becomes possible to identify the specific characteristic modes that are excited by track irregularities.

The practical application of Health Card requires that the detection parameters be set for specific wagon types on the basis of vehicle simulation and field observations. It is possible to extract car body accelerations or angular rates from vehicle simulation packages. Simulation test cases can be developed that produce vehicle responses that are considered to be borderline. The resulting acceleration traces can be used to tune the detection algorithms. The frequency selectivity of the method allows specific modes, say a roll mode in wagons with high centre of mass, to be specifically targeted.

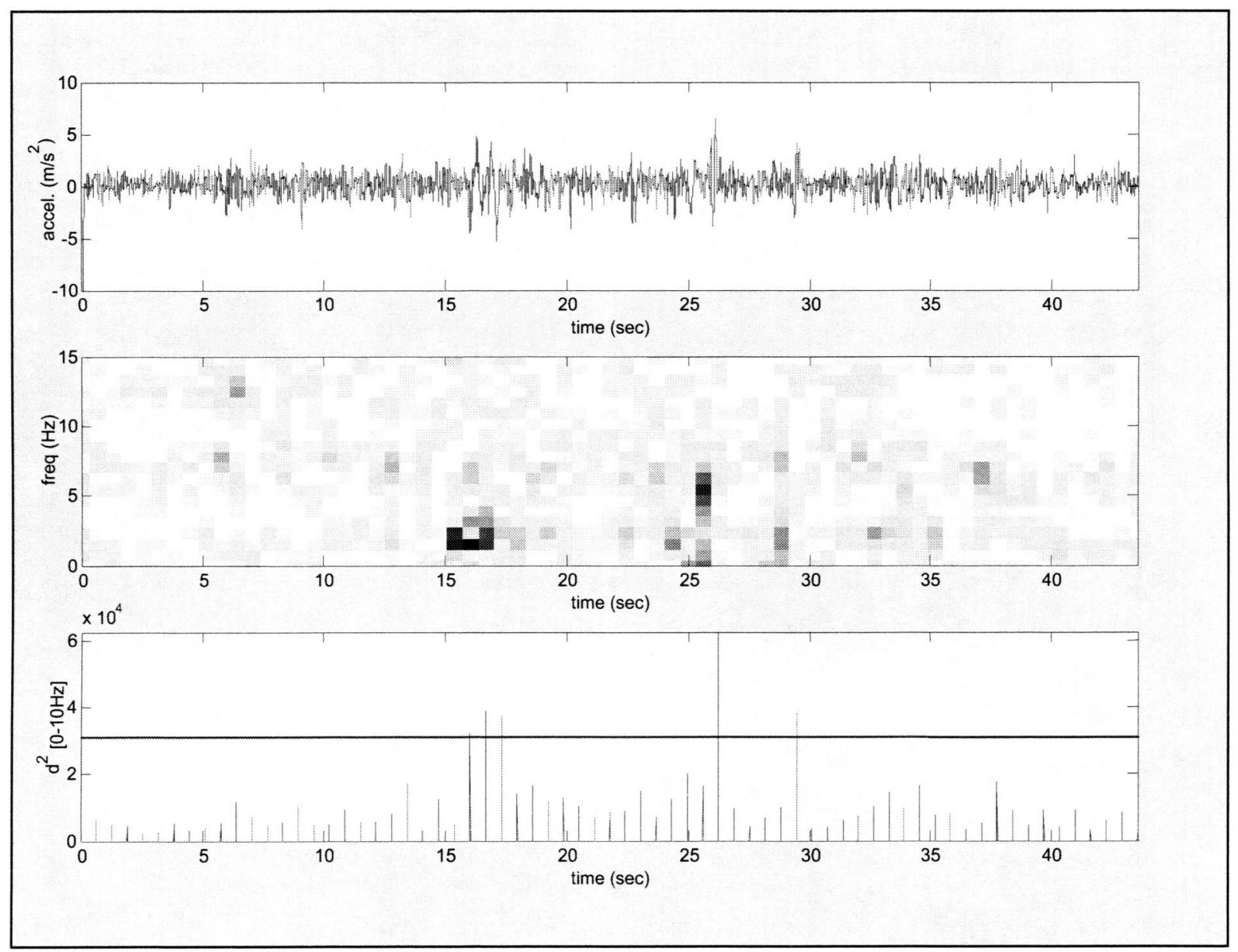

Figure 8. Time Series (top) and Spectrogram (centre) and Classification Output for Vertical Wagon Body Acceleration Signal.

\section{EXPERIMENTAL RESULTS}

Figure 9 shows a Queensland Rail gondola type wagon in a heavy rail vehicle testing facility at the Centre for Railway Engineering at Central Queensland University. This is an aluminum wagon with belly dump doors, designed for coal transport. The two wheel sets at one end of the vehicle are replaced by two hydraulically actuated beams. Each beam is lifted by two actuators allowing different vertical excitations to be applied to each end of each axle beam. This arrangement allows combinations of vertical bounce, roll and pitch to be produced. The wagon was ballasted at the end under test with
$30 \mathrm{~T}$ of scrap wheels and this produces an axle load reflective of the normal fully loaded condition. A set of Health Card transducers are mounted on this wagon. A typical arrangement for the radio and accelerometer unit is shown in Figure 10. Accelerations in each dynamic degree of freedom (i.e. Roll, Pitch, Yaw, Vertical, Lateral and Longitudinal) are calculated in the Health Card from the observed accelerations at three corners of the car body. These are weighted in terms of energy in the range of $0-10 \mathrm{~Hz}$ and the result is reported to the locomotive display unit, mounted on an adjacent laboratory bench, via the Bluetooth radio links. For these tests we did not 
allow the Health Cards to perform the classification task as the setting of classification thresholds is still under review.

A range of excitations was applied and the Health Cards were observed to be readily able to resolve the car body motions into six degrees of freedom. Figure 11 shows the results of a "roll" excitation of the car body as reported on a locomotive display developed for field trials with a consist of up to four gondola wagons with Health Cards. The row numbers $1,2,3$ \& 4 represent the four wagons in the test train. The colors on the display give a visual sense of the relative magnitudes occurring. Small residual responses are seen in the pitch and yaw degrees of freedom. The wagon was not laterally constrained during the test and some small yaw motions were observed.

The numbers alongside the bands are the absolute minimum and maximum values received for the respective band in the period covered by the display (approximately one minute). These numbers will allow scale factors to be applied manually during further field trials.

\section{CONCLUSIONS}

This paper has presented a novel device for online analysis of vehicle body motion signals to classify the vehicle response to the track and to detect potential derailments. Two variations on the basic device have been developed. One uses a train-line for power and communications and an alternative device is wireless using solar power and spread spectrum radio communications to establish a communications path along the consist. Solid state accelerometers and/or angular rate sensors detect the full six degrees of freedom associated with car body motion.

The Health Card device uses the Fast Fourier Transform (FFT) to efficiently convert the signal into a time-frequency spectrograph so that events can be detected according to their short-term spectral content. A detection method was developed which results in a metric similar to the short term RMS value, however the method provides a set of coefficients to weight the calculation according to frequencies of interest. These would be selected to match the vehicle modal responses in the various degrees of freedom.

Future work is identified which includes tuning the frequency weightings to detect signals of interest. For the train-wire based device, which is more computationally capable, a range of more advanced algorithms are under development. These include the development of artificial neural networks to classify time frequency signatures, and comparison of various time frequency analysis techniques including Wavelet analysis.

\section{ACKNOWLEDGMENTS}

This work is supported by the Cooperative Research Centre for Railway Engineering and Technologies and the Centre for Railway Engineering at the James Goldston Faculty of Engineering and Physical Systems, Central Queensland
University. The authors would like to thank Mr Eryl Lynch, Rolling Stock Project Coordinator, Queensland Rail for arranging access to rolling stock for field trials.

Dr Colin Cole, Mr Bernard Jansen, Mr Bryan Power and Dr Fujie Xia at the Centre for Railway Engineering provided many valuable insights in the areas of vehicle dynamics and provided considerable assistance during the experimental phases.

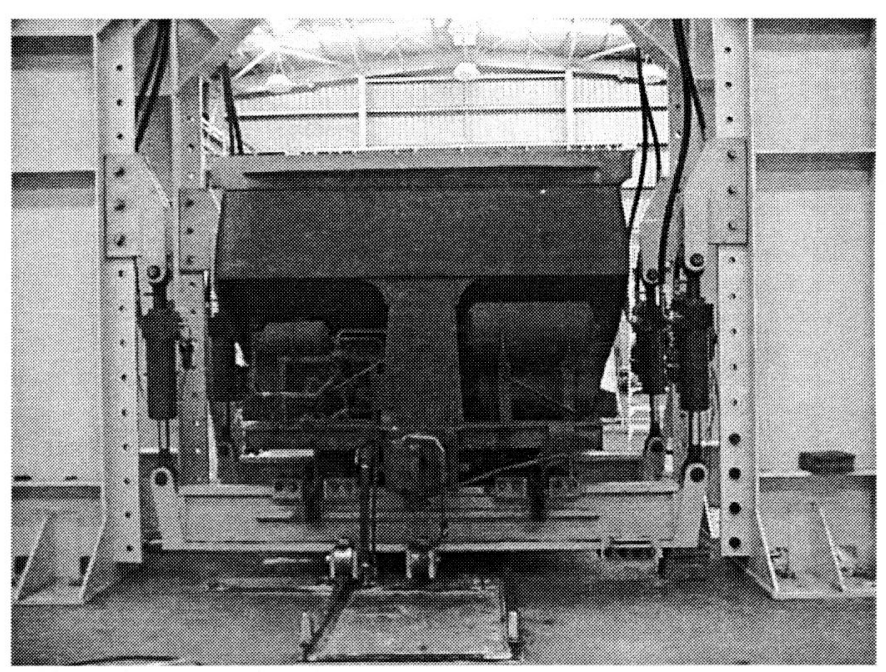

Figure 9. High Laboratory Test Rig Facility at the Centre for Railway Engineering, CQU.

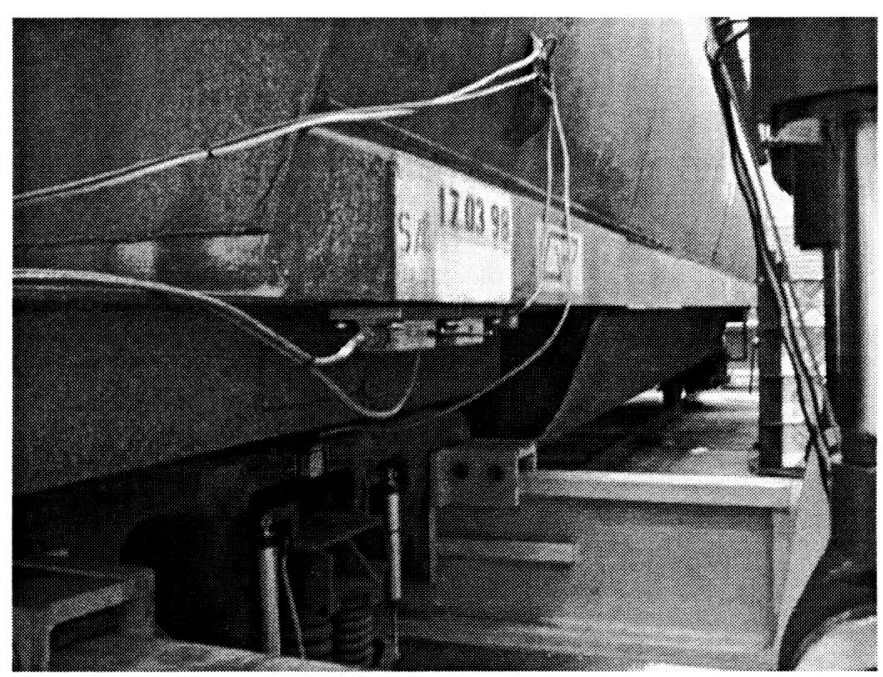

Figure 10. Health Card Accelerometer and Transmitter Mounted on Wagon 


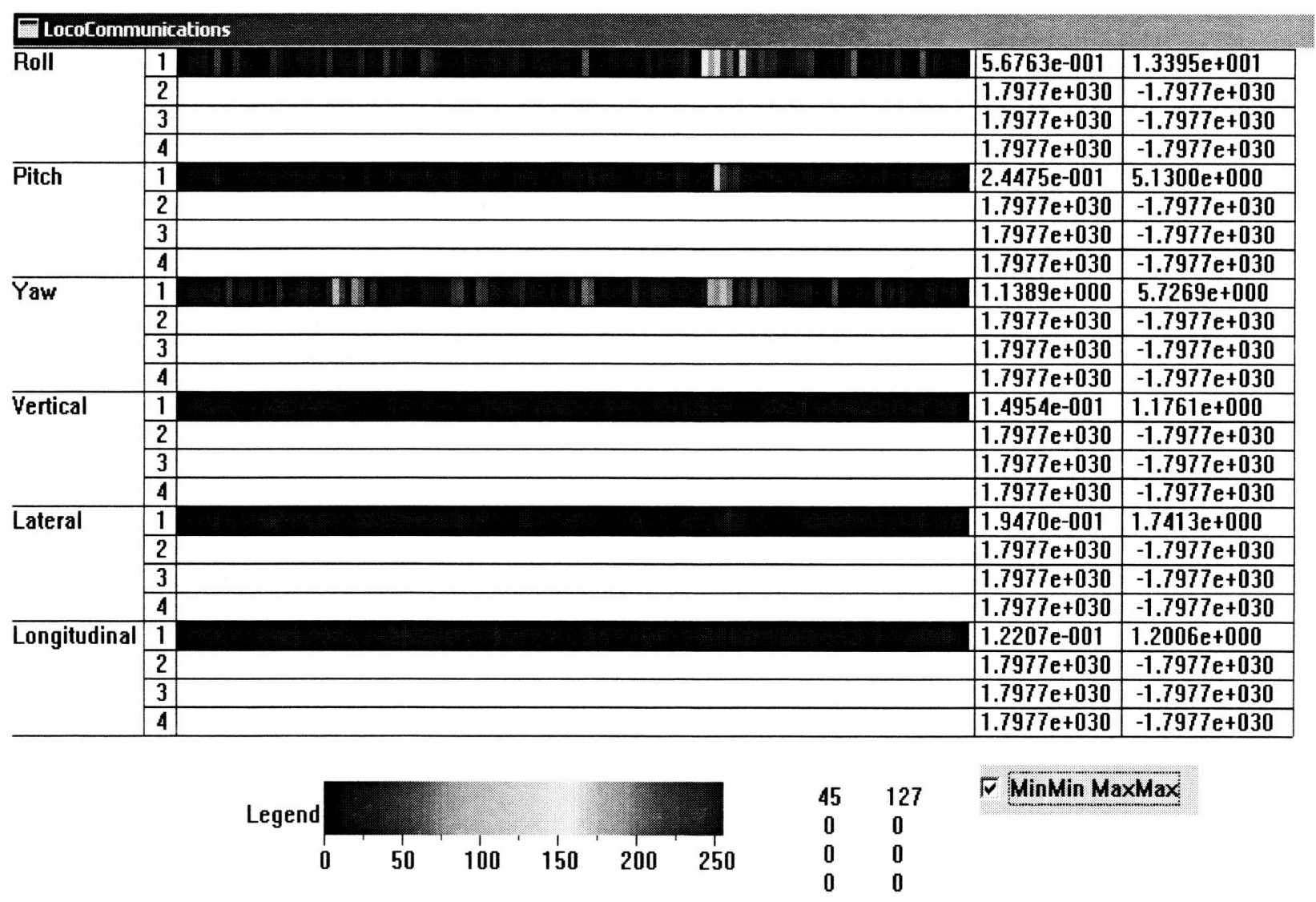

Figure 11. Display showing a Roll Event is from Wagon One.

\section{REFERENCES}

[1] Department of Transport and Regional Services (DTRS), "Code of Practice for the Defined Interstate Rail Network", Australia, 2003.

[2] Esveld, C.,"Modern Railway Track", 2nd ed., MRT Productions, Netherlands, 2001.

[3] Bonaventura C.S., Zarembski A.M., PaleseJ.W., "Tracksafe: A Track Geometry Car Based Real-Time Dynamics Simulator", Proceedings of the Joint Rail Conference, Pueblo, Colorado, 2005, pp 19-30.

[4] Li, D., T. Salahifar, J. Malone Jr. and S. F. Kalay, "Development of Performance-based Track Geometry Inspection", Proceedings 7th International Heavy Haul Conference, Brisbane, Australia, 10-14 June, 2001, pp 461-465. [5] McClanachan, M., M. Dhanasekar, D. Skerman, J. Davey, "Monitoring the Dynamics of Freight wagons", Conference on Railway Engineering, CORE 2002, Wollongong, pp 213-221.

[6] McClanachan, M., B.Scown, D. Roach, D. Skerman, B.Payne, "Autonomous Detection of Severe Wagon-Track Interaction Dynamics", $7^{\text {th }}$ International Heavy Haul Conference, 2001, pp 305-312.

[7] Cole, C., D. Roach, 1996, "Dynamic Response of Coal Wagons During Normal Operation", 1996 World Conference on Rail Research, Colorado Springs, pp 219-231.
[8] Ackroyd, P., S. Angelo and B. Nejikovsky, "Remote Ride Quality Monitoring of Acela Train Set Performance", Proceedings of the 2002 ASME/IEEE Joint Rail Conference, Washington, DC, April 23-25, 2002, pp 171-178.

[9] Federal Railroad Administration, "Track Safety Standards; Final Rule", Department of Transport:, 49CFR Part 213, June $22,1998$.

[10] Fry, K., "The Importance of Condition Monitoring in Providing Fault-Free Infrastructure, International Conference on Fault-Free Infrastructure, Effective Solutions to Improve Efficiency", IMechE Conference Transactions, 23-24 Nov 1999, Derby, UK, pp 17-26.

[11] Donelson J., Edwards M.C., Filkins M.H., Punwani S.K., Stewart M.F., Toth D.G., Zavis W.M., "Performance of an OnBoard System in Revenue Service Demonstration", Proceedings of the Joint Rail Conference, Pueblo, Colorado, 2005, pp 123129.

[12] Rieckenberg, T., "Study of Undetected Derailments in Europe", Track and Signal Magazine, 8, No.2, April-May-June 2004, pp 106-109.

[13] Echelon Corporation Power Line Smart Transceivers, http://www.echelon.com/products/transceivers/powerline/defaul t.htm 